\title{
Multi-boundary cardiac data visualization based on multidimensional transfer function with ray distance
}

\author{
Fei Yang ${ }^{\mathrm{a}}$, Lei Zhang ${ }^{\mathrm{b}}$, Weigang Lu ${ }^{\mathrm{c}, *}$, Wangmeng Zuo ${ }^{\mathrm{d}}$, Kuanquan Wang ${ }^{\mathrm{d}}$, Henggui Zhang ${ }^{\mathrm{d}}$ \\ and Yeming $\mathrm{Li}^{\mathrm{e}}$ \\ ${ }^{a}$ School of Mechanical, Electrical \& Information Engineering, Shandong University, Shandong, China \\ ${ }^{b}$ School of Art and Design, Harbin University, Heilongjiang, China \\ ${ }^{c}$ Department of Educational Technology, Ocean University of China, Shandong, China \\ ${ }^{d}$ School of Computer Science and Technology, Harbin Institute of Technology, Heilongjiang, China \\ ${ }^{e}$ Admissions Office, Harbin University, Heilongjiang, China
}

\begin{abstract}
A crucial role during the implementation of volume visualization is to identify the optimal transfer function, since the vital information and structure can be highlighted and revealed. The boundary of the volume is shared by respective portion of the two materials formed out of it, which causes undesirable thickening and ambiguity of the boundary explored via traditional LH (Low and High) histogram. To address this issue, initially a modified LH histogram construction method is introduced to intuitively and conveniently visualize cardiac volume for user interaction. Subsequently, the $f$-LH histogram is presented to further identify and visualize each portion of the boundary accurately. An appropriate multidimensional transfer function generation is proposed by using variables in $f$ - $\mathrm{LH}$ space and spatial information, for visualizing the multi-boundary cardiac volume data.
\end{abstract}

Keywords: Interactive visualization, partial effect, multidimensional transfer function, multi-boundary data

\section{Introduction}

Visualization techniques have been thoroughly investigated in exploring the complex 3D dataset, especially for medical applications. Transfer function plays a vital role during visualization, since it performs the mapping between voxels and their optical properties. Generation of transfer function thus plays a dominant role in the final rendering results, which directly affect the user's perception of the volume of data [1].

Simplest way to arrive at a desired transfer function for user is to achieve it through trial-and-error modification. Bajaj et al. [2] presented a data-centric approach which made use of contour spectrum to assist users to determine uniquely shaped iso-surfaces, which helps to determine the features in the final visualizing results.

\footnotetext{
${ }^{*}$ Corresponding author: Weigang Lu, Department of Educational Technology, Ocean University of China, Shandong, China. Tel./Fax: 086-0532-66787168; E-mail: luweigang@ouc.edu.cn.
}

0959-2989/14/\$27.50 @ 2014 - IOS Press and the authors. 
With the increase in features of interest hide in the volume of data, 1D transfer function is not sufficient to reveal enough information in the dataset. Levoy [3] found the gradient value of voxel could provide additional information for transfer function generation and extended the transfer function to the second dimension, i.e., voxel intensity and voxel gradient. Important information, such as the spatial relationship, the size and the shape of different materials, are carried by boundaries. Kindlmann and Durkin [4] first introduced the second order derivative of voxel, and created a 2D histogram. Boundaries presented as arches in the histogram and can be distinguished subsequently.

Recently, there is an increasing trends to combine user interactions into visualization pipeline in order to facilitate the inspection of volume of data with manipulating visualization for many applications. Kniss et al. [5] derived a set of convenient widgets to provide direct manipulation for intuitive exploring boundaries. Zhang et al. developed a platform integrating multivolume visualization method for both heart anatomical data and electrophysiological data visualization [6-8]. Wang et al. [9] added a new ingredient to the context-preserving model to fit the volume of human heart dataset on the GPU. Zhang et al. proposed a novel approach for revealing detailed structures in the human heart anatomy via perception-based lighting enhancement [10].

The objective of this paper is to improve visualization quality of multi-boundary cardiac volume. In Section 2, we have explained how to construct a simplified LH histogram and the method of generating the improved $f$-LH histogram is also described. In Section 3 , boundary values in $f$-LH histogram are used in combination with spatial information to design multidimensional transfer function for multi-boundary cardiac volume of data. Details of the experimental results are provided in Section 4. Finally, conclusions of the work are presented in Section 5.

\section{Modified LH histogram construction}

Šereda [11] suggested the conventional LH Histogram as a 2D histogram in which two axes stand for $F L$ and $F H$, respectively. The two variables denote two extra properties for each voxel. For the materials forming the boundary that the voxel belongs to, $F L$ is labeled as the value of material having the lower intensity and $F H$ is labeled as the higher value.

\subsection{Simplified LH histogram for segmented data}

Since the bias and noise have been filtered in segmented dataset, a technique is presented here for creating a simplified LH histogram. Tracking is made by simply searching for the $F L$ and $F H$ value among the neighborhood of the sample voxel. First, each sample voxel is labeled as interior or exterior via comparing it with its certain neighbors, which can speed up the searching time significantly. If current sample voxel intensity is the same as that of its all neighborhoods, the voxel is then marked as an inner voxel; otherwise the voxel is determined on the boundary.

For interior voxels, similar to LH histogram, both $F L$ and $F H$ values are denoted by their intensity values. The two values of exterior voxels are evaluated only by using neighbors of the voxel. The largest appeared intensity value of those neighbors is labeled as $F L$ or $F H$, which depends on whether the value is lower or higher than the intensity of current voxel. Evaluation of the $F L$ value and $F H$ value is given in Eq. (1): 


$$
F L=\left\{\begin{array}{ccc}
f\left(x_{i}\right) & x_{i} \in S_{\text {inner }} \\
\min \left\{f\left(x_{i}\right), f_{\text {hfreq }}\left(x_{i}\right)\right\} & x_{i} \in S_{\text {boundary }}
\end{array} \quad F H=\left\{\begin{array}{cc}
f\left(x_{i}\right) & x_{i} \in S_{\text {inner }} \\
\max \left\{f\left(x_{i}\right), f_{\text {hfreq }}\left(x_{i}\right)\right\} & x_{i} \in S_{\text {boundary }}
\end{array}\right.\right.
$$

where $x_{i}$ means a voxel in the volume, and $f\left(x_{i}\right)$ denotes the intensity of $x_{i} . S_{\text {inner }}$ and $S_{\text {boundary }}$ are the set of voxels inside the materials and on the boundaries respectively. $f_{h f r e q}\left(x_{i}\right)$ means the highest appeared intensity of neighbors of current voxel $x_{i}$.

Figure 1 demonstrates original cardiac slice data and the corresponding simplified LH histogram. As shown in Figure 1(b), one single point above the diagonal of the simplified LH histogram represents a boundary formed by two heart tissues. Points on the diagonal are the accumulation and distribution of voxels inside the tissues. In Figure 1(b), voxels with the highest value in the histogram correspond to the background. As indicated by the highlighted pink line, every boundary constituted by a tissue and the background is projected to a point on this line. Contour of the whole heart can then be visualized by selecting all the points on this line in the histogram as shown in Figure 1(c).

\subsection{Improved f-LH histogram}

Although the boundary between materials can be visualized via the LH histogram, exploring each side of the boundary distinctly is still a difficult task. Boundaries are often artificially thickened in the visualization result. It is a fact that two materials share a boundary, while boundary voxels of each material are often falsely classified to the same portion, which is shown clearly in Figure 2. In Figure 2(a), the boundaries formed by the background and left ventricle are denoted by red colored line, where as the boundary is differentiated accurately in Figure 2(b). The pink profile, i.e. the outside layer of the left ventricle-background boundary is the background portion, where as the red profile, which appears as the inner layer of the boundary, indicates the left ventricle portion.

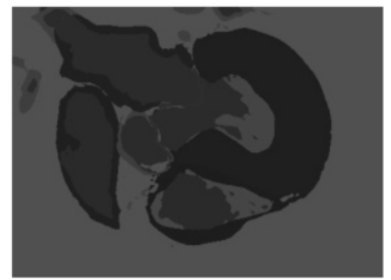

(a)

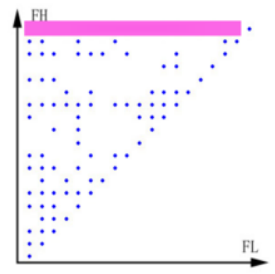

(b)

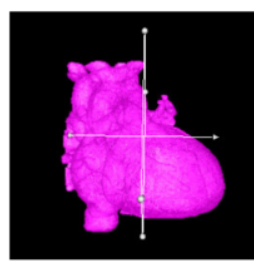

(c)

Fig. 1. Cardiac dataset and the corresponding histogram. (a) Slice data, (b) Simplified LH histogram and (c) Contour of the whole heart.

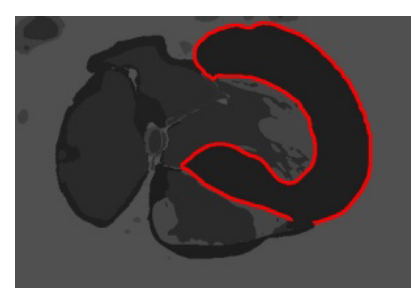

(a)

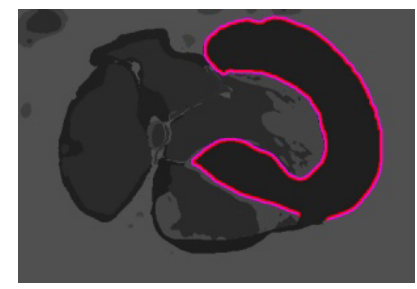

(b)

Fig. 2. The boundary formed by the background and left ventricle in the slice data. (a) Two portions of the boundary not differentiated and (b) The differentiated two portions of the boundary. 
To distinguish the two boundary portions, the simplified LH histogram is improved. In stead of $F L$ and $F H$, in the $f$-LH space, one of domain $L o H$ (Low or High) is defined as the $F L$ or $F H$ value which is detected in the simplified LH space, and the other domain $f$ is defined as the voxel intensity. The value of $f$ and $L o H$ can be obtained using Eq. (2):

$$
f=f\left(x_{i}\right) \quad L o H=\left\{\begin{array}{cc}
f\left(x_{i}\right) & x_{i} \in S_{\text {inner }} \\
f_{\text {hreq }}\left(x_{i}\right) & x_{i} \in S_{\text {boundary }}
\end{array}\right.
$$

Estimated voxel is directly projected onto the $f$-LH histogram without comparing its $f$ value with $\mathrm{LoH}$ value and swapping them as done for generating the simplified LH histogram. The improved $f$ LH histogram is thus created. Figure 3 demonstrates the LH histogram and the corresponding visualization result of the ventricle-background boundary. The $f$-LH histogram and its corresponding result are also demonstrated in Figure 3.

In Figure 3(a), points on vertical red line with $F L$ value of 31 and the $F H$ value greater than 31 represent boundaries formed by left ventricle with the intensity value of 31 and those tissues with the intensity greater than 31 . The isolated red point means the left ventricle-right ventricle boundary. Thus all the red points are the projection of the boundaries formed by left ventricle and other tissues of heart. Similarly, the horizontal pink line demonstrates all boundaries formed by the background and other tissues of heart. The green dot located at the intersection of the pink line and red line corresponds to the voxels on the left ventricle-background boundary. However, the voxels in these two sides of this boundary are projected to the same point. By this way, the two portions are denoted by same color using the transfer function based on the simplified LH histogram, which are marked as red color in Figure 3(a). As a result, the left ventricle side of the boundary is artificially thickened and two portions of the boundary cannot be properly differentiated.

In Figure 3(c), each point $p$ in $f$-LH histogram represents the material $A$ where $p$ belongs to the boundary formed by $A$ and the other material $B$. Then left ventricle portion in each boundary between left ventricle and a certain tissue are projected as a red point with $f$ value of 31 in the $f$-LH histogram. In the same way, background portion in each boundary are projected as a pink point with $f$ value of 80 . The green dot in Figure 3(a) is thus divided into two dots, the yellow dot and the blue dot, in Figure $3(\mathrm{c})$. By selecting the yellow dot in the $f$-LH histogram, the left ventricle portion of the left ventriclebackground boundary is exactly identified and colored by red, while the background side is colored by pink. The two portions are thus differentiated clearly without any artificial thickening or ambiguity. The magnified visualization result based on the simplified LH histogram is shown in Figure 3(b) and the result based on the $f$-LH histogram is displayed Figure 3(d).

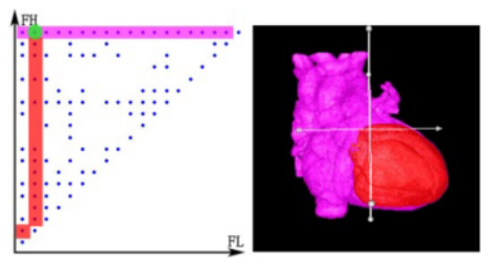

(a)

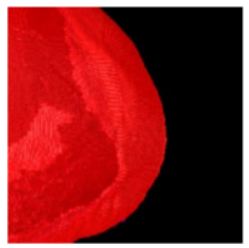

(b)

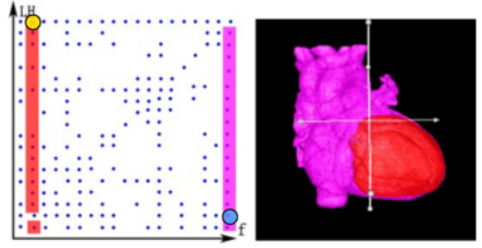

(c)

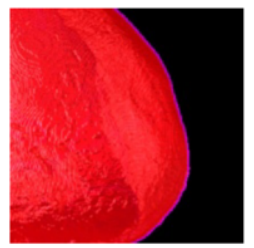

(d)

Fig. 3. The boundary formed by the background and left ventricle. (a) LH histogram and visualization result, (b) Magnified result of (a), (c) f-LH histogram and visualization result and (d) Magnified result of (c). 


\section{Multidimensional transfer function generation for multi-boundary cardiac visualization}

Multidimensional transfer function generation is beneficial and meaningful for multi-boundary cardiac visualization by including different variables into domains of transfer function. Each voxel in the dataset has scalar values including intensities of the voxel, as well as geometry space information. By combining the properties of these data sets in the multidimensional transfer functions, interesting features in dataset can be properly identified. The opacity in the transfer functions is given in Eq. (3):

$$
\operatorname{Opac}(x, y, z)=\operatorname{Opac}_{f-L H}(x, y, z) \cdot \operatorname{Opac}_{\text {Space }}(x, y, z)
$$

Where $\operatorname{Opac}_{f-L H}(x, y, z)$ is acquired through the $f$-LH histogram interface. $O p a c_{\text {Space }}(x, y, z)$ is computed by incorporating the $f$-LH space into the space information of the voxel, which is indicated in Eq. (4):

$$
\operatorname{Opac}_{\text {Space }}(x, y, z)=\frac{\sqrt{\left(x-X_{\text {in }}\right)^{2}+\left(y-Y_{\text {in }}\right)^{2}+\left(z-Z_{\text {in }}\right)^{2}}}{\sqrt{\left(X_{\text {out }}-X_{\text {in }}\right)^{2}+\left(Y_{\text {out }}-Y_{\text {in }}\right)^{2}+\left(Z_{\text {out }}-Z_{\text {in }}\right)^{2}}} \cdot\left\|T_{\text {Rap Dis }}\right\|
$$

where $(x, y, z),\left(X_{\text {in }}, Y_{\text {in }}, Z_{\text {in }}\right)$ and $\left(X_{\text {out }}, Y_{\text {out }}, Z_{\text {out }}\right)$ are the coordination of the current voxel, sample voxel on the inner and outer boundary along the ray casting through the volume respectively. The inner and outer boundaries are previously identified through $f$-LH histogram. $\left\|T_{\text {Ragis }}\right\|$ denotes the distance of the ray casting through the material that current voxel belongs to.

\section{Experimental results}

In this section, the data sets of female heart from the Visible Human Project are considered to evaluate the performance of the proposed method. Figure 4(a) shows the simplified LH histogram, and the visualization result is shown in the right of Figure 4(a). Vertical and horizontal yellow lines in the histogram corresponds all boundaries formed by right atrium with the intensity of 32 and other adjacent tissues with the intensity greater or less than 32 . Subsequently, the whole right atrium contour can be visualized by selecting all the yellow points in the histogram. The single cyan point on the diagonal means right atrium-ventricle valve, and this semi-transparent tissue is visualized conveniently and intuitively via the simplified LH histogram interface. Boundaries between aorta with the intensity value of 62 and its several important adjacent tissues are selectively visualized in Figure 4(b). The aortaright ventricle boundary is colored in red. The green, blue and yellow boundary is aorta-right atrium, aorta-vena cava and aorta-pulmonary trunk boundary, respectively. Interior of aorta with the intensity of 44 is colored in cyan. 


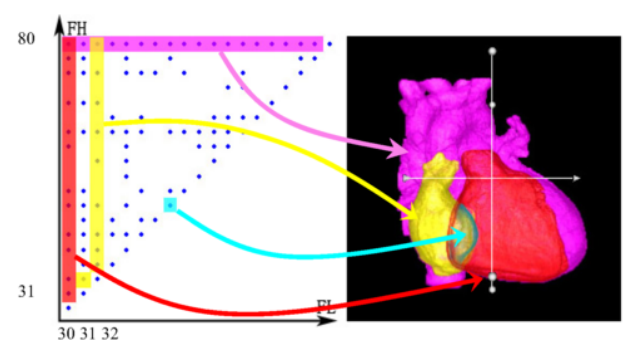

(a)

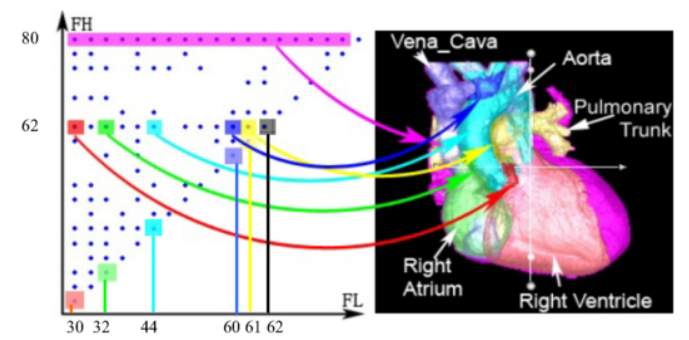

(b)

Fig. 4. Visualizing various tissue boundaries of the heart based on the simplified LH histogram. (a) Right ventricle-right boundary and contours of whole heart and (b) Boundaries formed by aorta and those tissues adjacent to it.

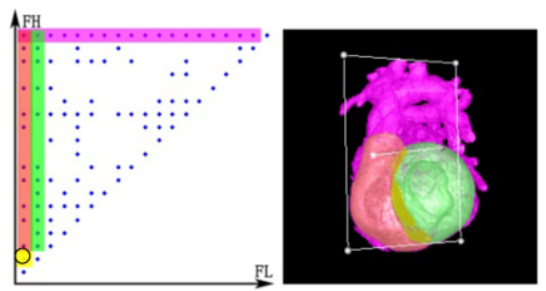

(a)

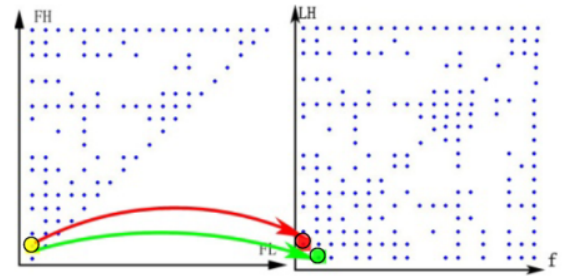

(b)

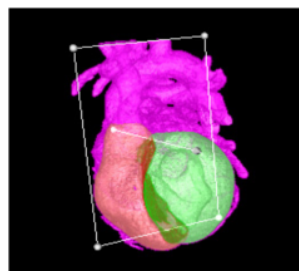

(c)

Fig. 5. Distinct visualization result of each side of the left ventricle-right ventricle boundary via f-LH histogram. (a) The boundary visualized using the simplified LH histogram, (b) Improved f-LH histogram and (c) Each side of the boundary visualized using f-LH histogram.

Figure 5(a) shows the left ventricle-right ventricle boundary which is colored yellow using the transfer function based on the simplified LH histogram. Voxels in the two portions on this boundary are all projected to the same yellow dot, and the two portions are consequently colored as same (yellow), as shown in the right of Figure 5(a). This results in false thickening of boundary. After improving the simplified LH histogram, two symmetry dots, i.e. the red dot and green $\operatorname{dot}$ in $f$-LH histogram replace the yellow dot in the simplified LH histogram, as shown in Figure 5(b). The red and the green dot mean the right ventricle and left ventricle side of the left ventricle-right ventricle boundary respectively. The corresponding Semi-transparent right ventricle portion (red) and left ventricle portion (green) are possible to be accurately detected and exactly visualized in Figure 5(c).

Figure 6 shows the visualization result of various tissues of heart. All boundaries are identified and distinguished by ways of the generated multi-dimensional transfer function for multi-boundary cardiac data. Tissues are differentiated, and size, shape and structure of each tissue are explored. Since spatial information is added as a dimension of multi-dimensional transfer function, spatial relation among tissues, for example right ventricle (red) and its neighbor tissues, left ventricle (blackish green), right atrium (purple) and aorta (yellow), can be observed clearly. Due to the consideration of distance of the ray casting through the material in the multi-dimensional transfer function, mutual occlusion of various tissues is eliminated. As shown in Figure 6, left ventricle (blackish green) occluded by right ventricle (red), as well as right atrium-ventricle valve (green) hiding between right ventricle (red) and right atrium (purple) are distinctly revealed with current viewpoint. 


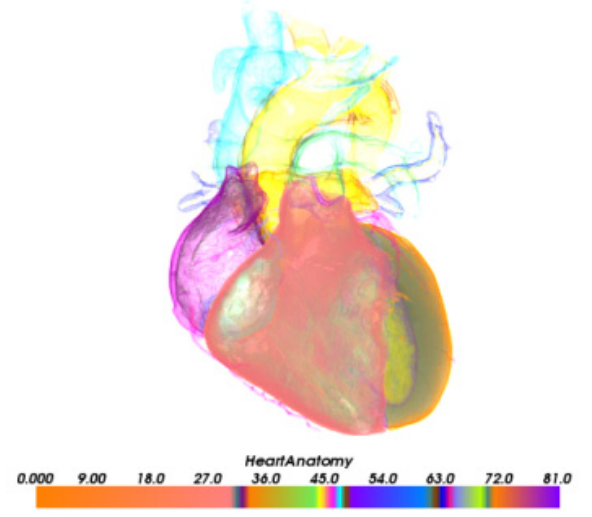

Fig. 6. Visualization result of various heart tissues based on the multi-dimensional transfer function.

\section{Conclusion}

The main aim of the paper is to develop an effective method for multidimensional transfer function generation to visualize the multi-boundary cardiac volume easily and intuitively. In comparison to previous methods, our proposed method explores the features interactively and distinctly.

In order to interactively visualize the multi-boundary of heart, a simplified LH histogram is introduced for designing the transfer function. Using the proposed improved $f$-LH histogram on the basis of the simplified LH histogram, general partial effect arisen on the boundary is eliminated, and each material portion of the boundary is exactly distinguished. This $f$-LH histogram model provides a way for easier identification and selection of exact boundaries for the users.

The multidimensional transfer function for the cardiac data is modulated by the function of the variables of $f$-LH space and spatial position of the tissue. The approach facilitates and improves exploring features of interest in the cardiac data. User only needs to specify the interesting structure via the $f$-LH histogram. Anatomical structure features and inspection of internal detail with viewpoint variation are possible to be emphasized and observed intuitively.

\section{Acknowledgement}

The work is partially supported by the National Natural Science Foundation of China (NSFC) under Grant Nos. 61173086, Scientific Research Foundation of Shandong University under Grants No. 1050513200007, the Fundamental Research Funds for the Central Universities No. 201413012, Youth Foundation of Harbin University under Grants No. HUYF2013-025 and Doctoral Fund of Harbin University under Grants No. HUDF2014-008.

\section{References}

[1] F. Zhou, X. Fan and B. Yang, Prospects and current studies on designing transfer function in volume rendering, Journal of Image and Graphics 13 (2008), 1034-1047.

[2] C.L. Bajaj, V. Pascucci and D. Schikore, The contour spectrum, Proc. IEEE Visualization, 1997, 167-174.

[3] M. Levoy, Display of surfaces from volume data, IEEE Computer Graphics and Applications 8 (1988), 29-37. 
[4] G. Kindlmann and J.W. Durkin, Semiautomatic generation of transfer functions for direct volume rendering, Proc. Volume Visualization, 1998, 79-86.

[5] J. Kniss, G. Kindlmann and C. Hansen, Interactive volume rendering using multidimensional transfer functions and direct manipulation widgets, Proc. IEEE Visualization, 2001, 255-262.

[6] L. Zhang, K.Q. Wang, W.M. Zuo and C.Q. Gai, G-Heart: A GPU-based system for electrophysiological simulation and multi-modality cardiac visualization, Journal of Computers 9 (2014), 360-367.

[7] L. Zhang, K.Q. Wang, W.M. Zuo and M.Z. Yang, Real-time multi-volume rendering for 3D electrophysiological data visualization based on graphics processing unit, ICIC Express Letters, Part B: Applications 4 (2013), 1625-1630.

[8] L. Zhang, K.Q. Wang, W.M. Zuo, Y.F. Wu and D.C. Han, GPU-based fusion method for 3D electrophysiological data visualization, Proc. International Conference on Computerized Healthcare, 2012, 51-56.

[9] K.Q. Wang, L. Zhang, C.Q. Gai and W.M. Zuo, Illustrative visualization of segmented human cardiac anatomy based on context-preserving model, Computer in Cardiology, 2011, 485-488.

[10] L. Zhang, K.Q. Wang, H.Z. Zhang, W.M. Zuo, X.Q. Liang and J. Shi, Illustrative cardiac visualization via perceptionbased lighting enhancement, Journal of Medical Imaging and Health Informatics 4 (2014), 312-316.

[11] P. Šereda, A. Vilanova, I.W.O. Serlie and F.A. Gerritsen, Visualization of boundaries in volumetric datasets using LH histograms, IEEE Trans. Visualization and Computer Graphics 12 (2006), 208-218. 